\title{
On-Site Coulomb Repulsion and Resonant Tunneling
}

\author{
Tai Kai $\mathrm{Ng}$ and Patrick A. Lee \\ Department of Physics, Massachusetts Institute of Technology, Cambridge, Massachusetts 02139
}

(Received 9 May 1988)

\begin{abstract}
We study the effect of on-site Coulomb repulsion on the process of resonant tunneling. We find that the tunneling peak results from a crossover from the high-temperature Kondo phase to the lowtemperature mixed-valence phase of the system when the chemical potential is varied across the on-site localized-state energy. Consequently, the line shape is non-Lorentzian, with rather unusual temperature dependence. Moreover, a magnetic field does not split the tunneling peak, but the line shape is modified. The effect of coupling between localized states is also discussed.
\end{abstract}

PACS numbers: $71.55 . \mathrm{Jv}, 73.40 . \mathrm{Qv}$

In this Letter we discuss the effect of intra-atomic Coulomb interaction on the resonant site in the process of resonant tunneling. Resonant tunneling is thought to be the dominating mechanism for conduction at very low temperature through small systems with localized states. ${ }^{1,2}$ In the noninteracting case, this mechanism has been discussed by a number of authors ${ }^{3-5}$ and the phenomenon can be described by a 1D model Hamiltonian $H_{0}$, $H_{0}=-t \sum_{\sigma= \pm 1}\left\{\sum_{i \geq 1}\left(c_{i \sigma}^{\dagger} c_{i+1 \sigma}+\right.\right.$ H.c. $)+\sum_{i \leq-2}\left(c_{i \sigma}^{\dagger} c_{i+1 \sigma}+\right.$ H.c. $\left.)\right\}-\tilde{t}_{R} \sum_{\sigma}\left(c_{0 \sigma}^{\dagger} c_{1 \sigma}+\right.$ H.c. $)-\tilde{t}_{L}\left(c_{-1 \sigma}^{\dagger} c_{0 \sigma}+\right.$ H.c. $)+\epsilon_{0} \sum_{\sigma} n_{0 \sigma},(1)$

where $i$ is the site index, $\sigma= \pm 1$ is the spin index, and $n_{0 \sigma}$ is the number operator for electrons with spin $\sigma$ on the site $i=0$ with on-site energy $\epsilon_{0}$. The parameters $\tilde{t}_{L}$ and $\tilde{t}_{R} \ll t$ simulate the "potential barrier" in the resonant transmission problem where the site $i=0$ simulates the resonance region with energy $\epsilon_{0}$. In realistic systems, the $i=0$ site represents a localized state at energy $\epsilon_{0}$ in a disordered region with localization length $l_{0}$, with $\tilde{t}_{L(R)} \sim t \exp \left(-l_{L(R)} / l_{0}\right)$, where $l_{L(R)}$ is the distance between localized state and the left or right electrode. At most energy values a particle is almost totally reflected by the potential barrier, but in a very small energy interval of width $\Gamma_{m}$ around $\epsilon_{0}$, the particle transmission is greatly enhanced.

In this Letter we shall study the effect of adding an interaction term $H_{I}=U n_{0 \nmid} n_{0 \downarrow}$ to the Hamiltonian $H_{0}$, with $U \sim e^{2} / l_{0}$ describing the effect of Coulomb interaction between two electrons of different spins on the localized state. Typically $U \gg \tilde{t}_{L}, \tilde{t}_{R}$. We shall also consider the effect of a magnetic field by adding an extra term $H_{B}=-\mu_{\mathrm{B}} B \sum_{\sigma} \sigma n_{0 \sigma}$ to the Hamiltonian. We note that this model is very similar to the one-impurity Anderson model with the impurity $d$ orbital replaced by a disorder-localized state and the conduction band replaced

by the left and right Fermi seas. The novel feature here is that the chemical potentials of the left and right Fermi seas can be adjusted separately.

We shall consider only the situation when the voltage difference between the two leads is small, ${ }^{1}$ so that we are in the linear-response regime. As a result, all we need to know is the dc conductance $G$ as a function of chemical potential $\mu$ for fixed $\epsilon_{0}$, or equivalently $G$ as a function of $\epsilon_{0}$ for fixed $\mu$. In this case, the tunneling process can be viewed as elastic scattering of electrons on the resonant site $^{6}$ and one can construct the $S$ matrix for the tunneling process in a way parallel to the work of Langreth ${ }^{7}$ for the 3D Anderson model. We first consider temperature $T_{0}=0$ where no elastic spin-flip scattering occurs, ${ }^{7}$ and we consider a spin- $\sigma$ tunneling electron, with energy $\epsilon_{k}$. Let the wave function of the electron be $u_{R \sigma} e^{i k x}$ $+v_{R \sigma} e^{-i k x}$ on the right-hand side of the scattering center and $u_{L \sigma} e^{i k x}+v_{L \sigma} e^{-i k x}$ on the left-hand side. Then the $S$ matrix defined by

$$
\left(\begin{array}{l}
v_{R \sigma} \\
u_{L \sigma}
\end{array}\right)=S_{\sigma}\left(\begin{array}{l}
u_{R \sigma} \\
v_{L \sigma}
\end{array}\right)
$$

is given by

$$
S_{\sigma}=\left(\begin{array}{cc}
1-2 \pi i \tilde{t}_{R}^{2} N_{\sigma}\left(\epsilon_{k}\right) G_{\sigma}\left(\epsilon_{k}+i \delta\right) & -2 \pi i \tilde{t}_{R} \tilde{t}_{L} N_{\sigma}\left(\epsilon_{k}\right) G_{\sigma}\left(\epsilon_{k}+i \delta\right) \\
-2 \pi i\left(\tilde{t}_{R} \tilde{t}_{L}\right) N_{\sigma}\left(\epsilon_{k}\right) G_{\sigma}\left(\epsilon_{k}+i \delta\right) & 1-2 \pi i \tilde{t}_{L}^{2} N_{\sigma}\left(\epsilon_{k}\right) G_{\sigma}\left(\epsilon_{k}+i \delta\right)
\end{array}\right),
$$

where $N_{\sigma}\left(\epsilon_{k}\right)$ is the spin- $\sigma$-component one-particle density of states of the noninteracting electron gas coupled to the resonant site. $G_{\sigma}(z)$ is the exact one-particle Green's function for an electron with spin $\sigma$ on the resonant site. The transmittance $T_{\sigma}$ for an electron with spin $\sigma$ is given by ${ }^{8}$

$$
T_{\sigma}\left(\epsilon_{k}\right)=\left|2 i \tilde{t}_{L} \tilde{t}_{R} N_{\sigma}\left(\epsilon_{k}\right) G_{\sigma}\left(\epsilon_{k}\right)\right|^{2}
$$


and the conductance can be related to the transmittances of electrons on the Fermi surface by the Landauer formula. ${ }^{9}$

At zero temperature only electrons on the Fermi surface, i.e., $\epsilon_{k}=\mu$, are important. In this case, the imaginary part of the exact self-energy of the Green's function $G_{\sigma}(\mu)$ is given by ${ }^{7}$

$$
\operatorname{Im} \Sigma_{\sigma}(\mu)=-\Gamma_{m}=-\pi\left(\tilde{t}_{L}^{2}+\tilde{t}_{R}^{2}\right) N_{\sigma}(\mu)
$$

and the exact occupation number $\left\langle n_{0 \sigma}\right\rangle=\left\langle c_{0 \sigma}^{\dagger} c_{0 \sigma}\right\rangle$ is ${ }^{7}$

$$
\left\langle n_{0 \sigma}\right\rangle=\pi^{-1} \operatorname{Im}\left[\ln G_{\sigma}(\mu+i \delta)\right] .
$$

Comparing Eqs. (4)-(6), we obtain

$$
T_{\sigma}(\mu)=\left(\frac{2 \tilde{t}_{L} \tilde{t}_{R}}{\tilde{t}_{L}^{2}+\tilde{t}_{R}^{2}}\right)^{2} \sin ^{2} \delta_{\sigma},
$$

where $\delta_{\sigma}=\pi\left\langle n_{0 \sigma}\right\rangle$. Equation (7) is important in that it allows us to obtain the conductance $G$ at zero temperature in terms of the exact ground-state occupation numbers $\left\langle n_{0 \sigma}\right\rangle$ as a function of $\epsilon_{0}$ (for fixed $\mu$ ). In particular, the maximum in $G$ (resonance) is obtained when $\delta=\pi / 2$, or when $\left\langle n_{0 \sigma}\right\rangle$ is $\frac{1}{2}$.

To solve our model Hamiltonian for the ground-state occupation number $\left\langle n_{0 \sigma}\right\rangle$ is equivalent to solving the ground state for the one-impurity Anderson model. The exact solution for the model is difficult to obtain but the qualitative behavior of the solution is well known. ${ }^{10-12}$ We shall first consider the situation of zero magnetic field. In this case, the ground state for the model is a spin singlet with $\left\langle n_{0 \uparrow}\right\rangle=\left\langle n_{0 !}\right\rangle=n_{0}\left(\epsilon_{0}\right)$. The qualitative behavior of $n_{0}\left(\epsilon_{0}\right)$ as a function of $\epsilon_{0}$ is as follows: Fixing the chemical potential $\mu$ at $\mu=0$, it can be shown from particle-hole symmetry that resonance $\left(n_{0}=\frac{1}{2}\right)$ is found exactly at $\epsilon_{0}=-U / 2$ (this is equivalent to saying that the Kondo resonance peak is exactly on the Fermi surface for the symmetric Anderson model) and that the conductance as a function of $\epsilon_{0}$ is symmetric around this point. Thus it is enough to study the case $\epsilon_{0}>-U / 2$. Now, let us consider the situation $-\Gamma_{m} \gg \epsilon_{0} \gg-U / 2$, where $\Gamma_{m}$ is the resonance linewidth in the noninteracting case. In this regime, the system is already in the Kondo limit and $n_{0}$ is very close to (but less than) $\frac{1}{2}$, which implies the existence of a large conductance. This is similar to the ordinary Kondo problem where a large resistance (or elastic scattering amplitude) is found at low temperature. The Kondo resonance moves away from the Fermi surface only when $\tilde{\epsilon}_{0} \rightarrow-\Gamma_{m}$, where $\tilde{\epsilon}_{0} \sim \epsilon_{0}+\Gamma_{m} / \pi \ln \left(U / \Gamma_{m}\right)$, when the system enters the mixed-valence regime, where $n_{0}$ decreases rapidly when $\tilde{\epsilon}_{0}$ crosses the Fermi surface, resulting in a rapid drop in conductance. The transmittance $T=T_{\uparrow}+T_{\downarrow}$ as a function of $\tilde{\epsilon}_{0}$ at zero temperature is shown in Fig. 1 (a) (solid line) with $U=200 \Gamma_{m}, \tilde{t}_{L}=\tilde{t}_{R}$, and $t=1000 \Gamma_{m}$, where $n_{0}$ is determined approximately by the Gutzwiller method ${ }^{13-15}$ applied to the one-impurity Anderson mod-

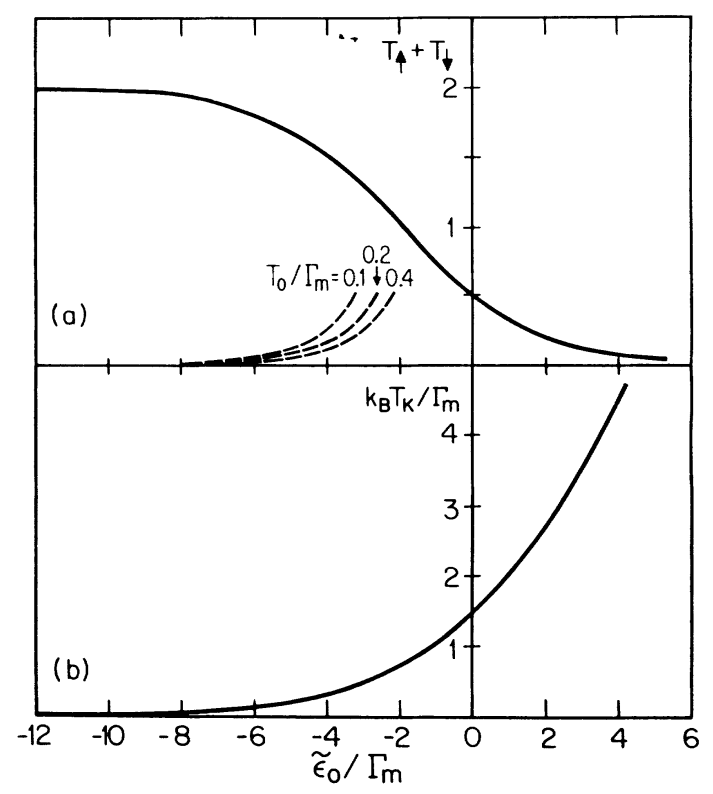

FIG. 1. (a) Transmittance as a function of $\tilde{\epsilon}_{0}$ for two situations: (i) zero-temperature result from the Gutzwiller method (solid line) and (ii) high-temperature expansion for three different temperatures (dashed lines) $k_{\mathrm{B}} T=0.1 \Gamma_{m}, 0.2 \Gamma_{m}$, and $0.4 \Gamma_{m}$; the leading logarithm series has been considered in our obtaining these results. (b) Kondo temperature $T_{\mathrm{K}}$ calculated from the Gutzwiller method as a function of $\tilde{\epsilon}_{0}$.

\section{el. Notice that $T_{\uparrow}+T_{\downarrow} \rightarrow 2$ in the Kondo limit.}

However, experiments are performed at finite temperature and the zero-temperature result is valid only when the temperature $T_{0}$ at which the experiment is performed is small compared with the characteristic excitation energy of the system, which in this case is the Kondo temperature $T_{\mathrm{K}}\left(\epsilon_{0}\right)$. A rough estimate for $T_{\mathrm{K}}\left(\epsilon_{0}\right)$ in the Kondo limit $\left(\epsilon_{0} \ll-\Gamma_{m}\right)$ is ${ }^{16} T_{\mathrm{K}}\left(\epsilon_{0}\right) \sim\left(U \Gamma_{m}\right)^{1 / 2} e^{-1 / J \rho}$ where $J \rho \sim 2 \Gamma_{m} / \pi\left|\epsilon_{0}\right|$. Notice that experimentally $\Gamma_{m} \sim 50 \mathrm{mK}$ and the Kondo temperature is unattainably low in this regime.

This estimate breaks down when $\tilde{\epsilon}_{0} \rightarrow-\Gamma_{m}$, when the system enters the mixed-valence regime where the characteristic temperature is $T_{\mathrm{K}}\left(\tilde{\epsilon}_{0}\right) \sim \Gamma_{m}$, and remains until $\tilde{\epsilon}_{0}>\Gamma_{m}$, when the system enters the "empty site" $\left(n_{0} \sim 0\right)$ regime where $T_{\mathrm{K}}\left(\epsilon_{0}\right) \sim \tilde{\epsilon}_{0}$. The characteristic temperature $T_{\mathrm{K}}\left(\tilde{\epsilon}_{0}\right)$ as a function of $\tilde{\epsilon}_{0}$ is shown in Fig. l(b) for the same set of parameters as used in Fig. 1(a), $T_{\mathrm{K}}\left(\tilde{\epsilon}_{0}\right)$ again being determined by the Gutzwiller method.

Typically, experiments are performed at a temperature lower than, but of the same order of magnitude as, $\Gamma_{m}$. Thus the zero-temperature result is applicable only when $\tilde{\epsilon}_{0}$ is in the range $\tilde{\epsilon}_{0} \gtrsim-\Gamma_{m}$ (mixed-valence regime). In particular, the large conductance in the Kondo limit is not expected to be observable. In fact, in this parameter range where $T_{0} \gg T_{\mathrm{K}}\left(\tilde{\epsilon}_{0}\right)$, a high-temperature perturbation series expansion for the transmittance is expected to 
yield the correct result. Proceeding as usual, we obtain the series expansion ${ }^{16,17}$

$$
T=\sum_{\sigma \sigma^{\prime}} T_{\sigma \sigma^{\prime}}=\left(\frac{2 \tilde{t}_{L} \tilde{t}_{R}}{\tilde{t}_{L}^{2}+\tilde{t}_{R}^{2}}\right)^{2}\left(\frac{\Gamma_{m}}{\epsilon_{0}^{*}}\right)^{2}\left(1+2(J \rho)^{*} \ln \left|\frac{\epsilon_{0}^{*}}{k_{\mathrm{B}} T_{0}}\right|+\cdots\right),
$$

where $(J \rho)^{*}=2 \Gamma_{m} / \pi\left|\epsilon_{0}^{*}\right| \quad$ and $\quad \epsilon_{0}^{*} \simeq \epsilon_{0}-\Gamma_{m} / \pi$ $\times \ln \left(\tilde{\alpha}\left|\epsilon_{0}^{*}\right| / U\right)$, with $\tilde{\alpha}$ a numerical factor of order 1 . The high-temperature expansion result for $T$ is shown in Fig. 1(a) (dashed line) for three different values of temperature $T_{0}$ (we approximate $\tilde{\alpha}=1$ ). Notice the rise in transmittance as $\tilde{\epsilon}_{0} \rightarrow-\Gamma_{m}$ when the temperature is lowered. The experimentally observed line shape is expected to be a smooth interpolation of the solid line (at $\left.\tilde{\epsilon}_{0} \gtrsim-\Gamma_{m}\right)$ and dashed line $\left(\tilde{\epsilon}_{0} \ll-\Gamma_{m}\right)$ in Fig. $1(\mathrm{a})$. The resulting line shape shows a peak in conductance as in the noninteracting case, but with a very different physical nature. In fact, it shows the crossover from the high- to low-temperature phase of the system as $\tilde{\epsilon}_{0}$ is varied. The line shape of this pseudoresonance is expected to be non-Lorentzian and asymmetric with respect to its peak position, and is temperature dependent only on the "high-temperature" side. The peak position can also be estimated roughly for a fixed temperature $T_{0}^{\prime}$ by identifying $T_{0}^{\prime} \sim T_{\mathrm{K}}\left(\tilde{\epsilon}_{0}^{\prime}\right)$ and its peak value can be estimated from the zero-temperature curve with $T_{\max }=T\left(\tilde{\epsilon}_{0}^{\prime}\right)$. The rather nonlinear shift in peak position as $T_{0}$ changes (in the range $T_{0}<\Gamma_{m}$ ) can be seen quite clearly from Fig. 1 (b).

The above picture changes completely when a strong magnetic field $\left(2 \mu_{\mathrm{B}} B \gg \Gamma_{m}\right)$ is applied, when the resonant site is spin polarized. In two dimensions, we align the field in the plane so that complications associated with orbital effects do not arise. ${ }^{18}$ As before, we shall first consider the zero-temperature situation. Consider the magnetic field pointing in the $-\hat{\mathbf{z}}$ direction so that the spin-down electron is lower in energy. Now consider $\epsilon_{0}$ changing from $\epsilon_{0} \sim \mu_{\mathrm{B}} B+\Gamma_{m}$ to $\mu_{\mathrm{B}} B-\Gamma_{m}$. Because the system is spin polarized, we expect $\left\langle n_{0 !}\right\rangle$ to increase from $\left\langle n_{0 !}\right\rangle \sim 0$ to $\left\langle n_{0 !}\right\rangle \sim 1$ in this region when $\epsilon_{0}$ decreases, while $\left\langle n_{0 \uparrow}\right\rangle$ stays close to zero; i.e., we expect to observe a real resonant peak in conductance in the spindown electron channel across this region, with $T_{\downarrow}(\max ) \sim 1$ while $T_{\uparrow} \sim 0$, by the Friedel sum rule. Notice that this is a one-particle resonant transmission as in the noninteracting situation, but not a Kondo resonance. The system stays polarized as $\epsilon_{0}$ decreases further, until it reaches the regime $\epsilon_{0} \lesssim-U$, when it becomes favorable energetically to place also a spin-up electron on the resonance site in forming the ground state. In other words, we expect that in the high-magnetic-field case, the main effect of the Coulomb interaction is to widen the spacing between the resonances in up- and down-spin channels from the "noninteracting" value of $2 \mu_{\mathrm{B}} B$ to $2 \mu_{\mathrm{B}} B+U$. Notice that because of the smallness of $\Gamma_{m}$ $(\sim 50 \mathrm{mK})$, it is in practice not difficult to reach this regime experimentally. Moreover, since these resonances are noninteractinglike, the characteristic excitation ener- gy of the system is always $T_{\mathrm{K}} \gtrsim \Gamma_{m}$, and the zerotemperature consideration would be a reasonable representation of the experimental situation $\left(T_{0}<\Gamma_{m}\right)$.

The position of the resonance peaks and line shapes can again be obtained approximately with the Gutzwiller method. ${ }^{19}$ Again it can be shown from combined particle $\rightarrow$ hole and $B \rightarrow-B$ symmetry that the transmittance as a function of $\epsilon_{0}$ is mirror symmetric around the point $\epsilon_{0}=-U / 2$. Therefore it is enough to consider the higher peak (resonance at $\epsilon_{0} \sim \mu_{\mathrm{B}} B$ ). In the limit $U \gg 2 \mu_{\mathrm{B}} B \gg \Gamma_{m}$, it can be shown that the resonant peak position is located at roughly $\epsilon_{0} \sim \mu_{\mathrm{B}} B-2 \Gamma_{m} / \pi$, with a resonance width of order $\Gamma_{m}$. As the magnetic field increases from zero, it is expected that the "crossover" peak will gradually turn into this "real" resonance peak. Thus in contrast to the noninteracting case, where a resonance is expected to split by $2 \mu_{\mathrm{B}} B$, here the resonance simply shifts and changes its line shape. The line shape is found to be strongly asymmetric around its peak position. The origin of this asymmetry can be seen from just the Hartree-Fock approximation where the renormalized on-site energy of the resonant site is given by $\epsilon_{R \sigma}=\epsilon_{0}-\sigma \mu_{\mathrm{B}} B+U\left\langle n_{0 \sigma}\right\rangle$, and (in the large-bandwidth limit)

$$
\left\langle n_{0 \sigma}\right\rangle=\pi^{-1} \tan ^{-1}\left(\Gamma_{m} / \epsilon_{R \sigma}\right) .
$$

It is straightforward to show that $\sin ^{2} \pi\left\langle n_{0 !}\right\rangle$ as a function of $\epsilon_{0}$ is not symmetric around the resonance point $\left\langle n_{0 !}\right\rangle=\frac{1}{2}$ (or $\epsilon_{0}^{\prime}=\mu_{\mathrm{B}} B-2 \Gamma_{m} / \pi$ ) in the limit $U \gg 2 \mu_{\mathrm{B}} B$ $\gg \Gamma_{m}$, by our determining $\epsilon_{0}$ as a function of $\left\langle n_{0 !}\right\rangle$ from Eq. (9). A plot of the transmittance as a function of $\epsilon_{0}$ around this resonance is given in Fig. 2 with $2 \mu_{\mathrm{B}} B=5 \Gamma_{m}$

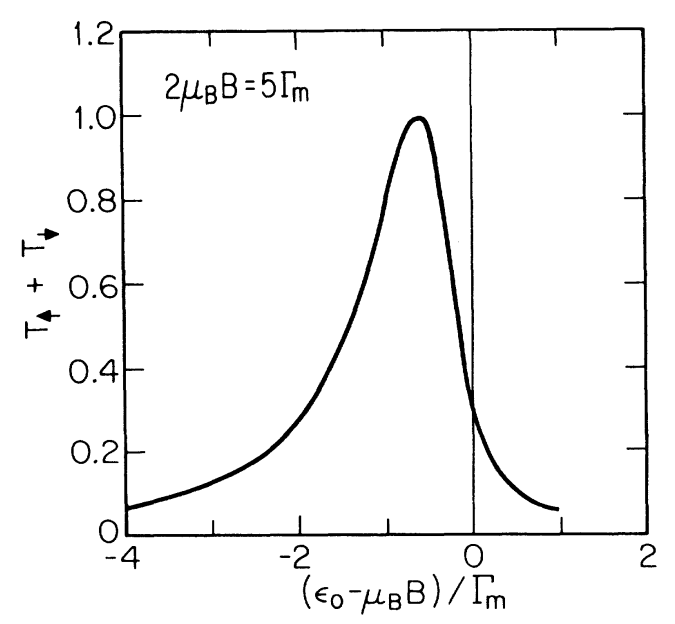

FIG. 2. Transmittance as a function of $\epsilon_{0}$ at zero temperature in the high-magnetic-field case, $2 \mu_{\mathrm{B}} B=5 \Gamma_{m}$. 
and the same set of parameters for $U$ and $t$.

Finally, we consider the interaction between different localized states that are singly occupied. It is known that on the localized side of the metal-insulator transition the local moments on singly occupied sites separated by distance $l_{2}$ interact via an antiferromagnetic exchange $J \propto \exp \left(-l_{2} / l_{0}\right)$. The exponential dependence on $l_{2}$ leads to a broad distribution of $J$, and at a given temperature $T_{0}$, the local moments with $J>T_{0}$ will form a spin singlet. ${ }^{20}$ The analysis that we presented so far ignores the coupling of the resonant site with other localized states and is valid provided that $J \ll \Gamma_{m}$. In the opposite limit $J \gg \Gamma_{m}$, the Kondo effect which affects the resonant tunneling when $T_{0} \lesssim \Gamma_{m}$ will be completely suppressed, because it is more favorable energetically to form a spin singlet with the other local moment than to form a Kondo singlet. In the energy range $\epsilon_{0} \ll 3 J / 4$, a stable spin singlet is formed between the two localized electrons which is essentially decoupled from the conduction band. For $\epsilon_{0} \gtrsim 3 J / 4$, the free spin on the other localized state is strongly screened by the formation of a virtual singlet with virtually excited electrons on state $\epsilon_{0}$, so that the Kondo effect is suppressed. The resulting "resonance" peak in transmittance shows the crossover between these two regimes as $\epsilon_{0}$ is varied. The line shape is nonLorentzian and is insensitive both to changes in temperature (for $\left.T_{0} \lesssim \Gamma_{m}\right)$ and magnetic field $\left(2 \mu_{\mathrm{B}} B \ll J\right)$, because the energy scale is now set by $J$, but not the Kondo temperature $T_{\mathrm{K}}$. Thus in order to compare with experiment, it is important to decide whether a given resonant structure is in the $J \gg \Gamma_{m}$ or $J \ll \Gamma_{m}$ regime. For example, if the length of the barrier can be reduced so that $l_{L}$ or $l_{R}$ is less than the typical separation between local moments, it becomes more likely to be in the $J \ll \Gamma_{m}$ Kondo-type regime.

In summary, we have studied the effect of Coulomb correlation in the process of resonant tunneling, using a $1 \mathrm{D}$, one-impurity site model. We have found that in the zero-magnetic-field case, the resonant transmission picture of one-particle tunneling is strongly modified. In particular, the resulting "pseudoresonance" peak in conductance describes the crossover from a high- to a lowtemperature phase of the system, as $\epsilon_{0}$ (or the chemical potential) is varied. The resulting conductance peak is expected to have a non-Lorentzian line shape, and with rather unusual temperature dependence. In the presence of a strong magnetic field $\left(2 \mu_{\mathrm{B}} B \gg \Gamma_{m}\right)$, the one-particle resonant transmission picture is found to be valid again.
However, the line shape of the resonant peak is found to be non-Lorentzian and asymmetric, as a result of Coulomb correlation. Furthermore, upon application of a magnetic field the peak simply shifts without any observable splitting by the Zeeman energy. We also point out that our description is quite sensitive to exchange coupling with other local moments which may exist in the experimental situation.

We acknowledge the support of the Joint Servies Electronics Program, Contract No. DAAL-03-86-K-0002.

${ }^{1}$ A. B. Fowler, G. L. Timp, J. J. Wainer, and R. A. Webb, Phys. Rev. Lett. 57, 138 (1986).

${ }^{2}$ S. J. Bending and M. R. Beasley, Phys. Rev. B 35, 2548 (1987)

${ }^{3}$ I. M. Lifschitz and V. Ya. Kirpichenkov, Zh. Eksp. Teor. Fiz. 77, 989 (1979) [Sov. Phys. JETP 50, 499 (1979)].

${ }^{4}$ M. Ya. Azbel, A. Harstein, and D. P. Divincenzo, Phys. Rev. Lett. 52, 1641 (1984).

${ }^{5}$ A. D. Stone and P. A. Lee, Phys. Rev. Lett. 54, 1196 (1985)

${ }^{6}$ A. Houghton, N. Read, and H. Won, Phys. Rev. B 35, 5123 (1987).

${ }^{7}$ D. C. Langreth, Phys. Rev. 150, 712 (1966).

${ }^{8}$ See, for example, E. Merzbacher, Quantum Mechanics (Wiley, New York, 1970).

${ }^{9}$ R. Landauer, Philos. Mag. 21, 863 (1970).

${ }^{10}$ H. R. Krishnamurthy, J. W. Wilkins, and K. G. Wilson, Phys. Rev. B 21, 1003,1004 (1980).

${ }^{11}$ See, for example, Theory of Heavy Fermions and Valence Fluctuations, edited by T. Kasuya and T. Saso, Springer Series in Solid-State Sciences Vol. 62 (Springer-Verlag, Berlin, 1985).

${ }^{12}$ See, for example, N. E. Bickers, Rev. Mod. Phys. 59, 845 (1987).

${ }^{13}$ M. C. Gutzwiller, Phys. Rev. 137, A1726 (1965).

${ }^{14}$ T. M. Rice and K. Ueda, Phys. Rev. Lett. 55, 995 (1985).

${ }^{15}$ C. M. Varma, W. Weber, and L. J. Randall, Phys. Rev. B 33, 1015 (1986).

${ }^{16}$ F. D. M. Haldane, Phys. Rev. Lett. 40, 416 (1978).

${ }^{17}$ J. Kondo, Prog. Theor. Phys. 32, 37 (1964).

${ }^{18}$ W. Xue and P. A. Lee, Phys. Rev. B 38, 3913 (1988).

${ }^{19}$ Notice that in the limit $U \gg 2 \mu_{\mathrm{B}} B \gg \Gamma_{m}$, the stable solution of the Gutzwiller approach is just the Hartree-Fock solution. See also T. Ogawa, K. Kanda, and T. Matsubara, Prog. Theor. Phys. 53, 614 (1975).

${ }^{20}$ R. N. Bhatt and P. A. Lee, Phys. Rev. Lett. 48, 344 (1982). 